\title{
A 2-Phase Traffic Model Based on a Speed Bound
}

\author{
Rinaldo M. Colombo ${ }^{1} \quad$ Francesca Marcellini $^{2} \quad$ Michel Rascle $^{3}$
}

\begin{abstract}
We extend the classical LWR traffic model allowing different maximal speeds to different vehicles. Then, we add a uniform bound on the traffic speed. The result, presented in this paper, is a new macroscopic model displaying 2 phases, based on a non-smooth $2 \times 2$ system of conservation laws. This model is compared with other models of the same type in the current literature, as well as with a kinetic one. Moreover, we establish a rigorous connection between a microscopic Follow-The-Leader model based on ordinary differential equations and this macroscopic continuum model.
\end{abstract}

2000 Mathematics Subject Classification: 35L65, 90B20

Keywords and phrases: Continuum Traffic Models, 2-Phase Traffic Models, Second Order Traffic Models

\section{Introduction}

Several observations of traffic flow result in underlining two different behaviors, sometimes called phases, see [10, 14, 16, 26. At low density and high speed, the flow appears to be reasonably described by a function of the (mean) traffic density. On the contrary, at high density and low speed, flow is not a single valued function of the density. This paper presents a model providing an explanation to this phenomenon, its two key features being:

1. At a given density, different drivers may choose different velocities;

2. There exists a uniform bound on the speed.

By "bound", here we do not necessarily mean an official speed limit. On the contrary, we assume that different drivers may have different speeds at

\footnotetext{
${ }^{1}$ Università degli Studi di Brescia, Via Branze 38, 25123 Brescia, Italy, Rinaldo.Colombo@UniBs. it

${ }^{2}$ Università di Milano-Bicocca, Via Bicocca degli Arcimboldi 8, 20126 Milano, Italy, F.Marcellini@Campus.Unimib.it

${ }^{3}$ Laboratoire de Mathematiques, U.M.R. C.N.R.S. 6621, Universitè de Nice, Parc Valrose B.P. 71, F06108 Nice Cedex, France, Rascle@Math.Unice.fr
} 

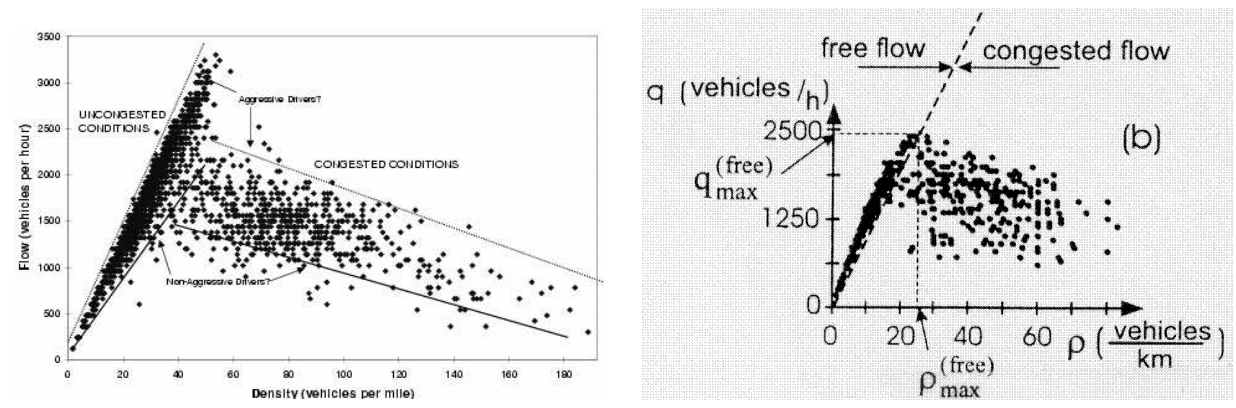

Figure 1: Experimental fundamental diagrams. Left, [28, Figure 1] and, right, [26, Figure 1], (see also [23]).

the same traffic density. Nevertheless, there exists a speed $V_{\max }$ that no driver exceeds. As a result from this postulate, we obtain a fundamental diagram very similar to those usually observed, see Figure 1 and Figure 2 , left. Besides, the evolution prescribed by the model so obtained is reasonable and coherent with that of other traffic models in the literature. In particular, we verify that the minimal requirements stated in [4, 13] are satisfied.

Recall the classical Lighthill-Whitham [32] and Richards 34] (LWR) model

$$
\partial_{t} \rho+\partial_{x}(\rho V)=0
$$

for the traffic density $\rho$. Assume that the speed $V$ is not the same for all drivers. More precisely, different drivers differ in their maximal speed $w$, so that $V=w \psi(\rho)$, with $w \in[\check{w}, \hat{w}], \check{w}>0$, being transported along the road at the mean traffic speed $V$. We identify the different behaviors of the different drivers by means of their maximal speed, see also [7, 8]. One is thus lead to study the equations

$$
\left\{\begin{array}{l}
\partial_{t} \rho+\partial_{x}(\rho v)=0 \\
\partial_{t} w+v \partial_{x} w=0
\end{array} \quad \text { with } \quad v=w \psi(\rho) .\right.
$$

Here, the role of the second equation is to let the maximal velocity $w$ be propagated with the traffic speed. Indeed, $w$ is a specific feature of every single driver, in other words is a Lagrangian marker. Therefore this model falls into the class of models introduced in [4], and later on extended in [30], see also [6, formula (1.2)].

Introducing a uniform bound $V_{\max }$ on the speed, we obtain the model

$$
\left\{\begin{array}{l}
\partial_{t} \rho+\partial_{x}(\rho v)=0 \\
\partial_{t} w+v \partial_{x} w=0
\end{array} \quad \text { with } \quad v=\min \left\{V_{\max }, w \psi(\rho)\right\}\right.
$$

We choose to reformulate the above quasilinear system in conservation form, similarly to [27, formula (1)], [5, formula (2.2)], [30, formula (1)], see also [36], 
as follows:

$$
\left\{\begin{array}{l}
\partial_{t} \rho+\partial_{x}(\rho v(\rho, \eta))=0 \\
\partial_{t} \eta+\partial_{x}(\eta v(\rho, \eta))=0
\end{array} \quad \text { with } \quad v(\rho, \eta)=\min \left\{V_{\max }, \frac{\eta}{\rho} \psi(\rho)\right\}\right.
$$

see the Remark 5.3 for further comments on this choice. This model consists of a $2 \times 2$ system of conservation laws with a $\mathbf{C}^{\mathbf{0}, \mathbf{1}}$ but not $\mathbf{C}^{\mathbf{1}}$ flow. Note in fact that $\eta / \rho=w \in[\check{w}, \hat{w}]$. A $2 \times 2$ system of conservation laws with a flow having a similar $\mathbf{C}^{\mathbf{0}, \mathbf{1}}$ regularity is presented in 21 and studied in [1].

From the traffic point of view, we remark that, under mild reasonable assumptions on the function $\psi$, the flow in (1.4) may vanish if and only if $\rho=0$, i.e the road is empty, or $\rho=R$, i.e. the road is fully congested. It is also worth noting the agreement between experimental fundamental diagrams often found in the literature and the one related to (1.4), see Figure 2, left.

From the analytical point of view, we can extend the present treatment to the more general case of a maximal speed $V_{\max }$ that depends on $\rho$, i.e. $V_{\max }=$ $V_{\max }(\rho)$. However, we prefer to highlight the main features of the model (1.4) in its simplest analytical framework.

As we already said, the model studied here, inspired from [10], falls into the class of "Aw-Rascle" models. So we could use the approach and the theoretical results of [3], which should apply here with minor modifications.

However, our approach is different: here, contrarily to the above reference, we establish directly a connection between the Follow-The-Leader model in Section 4 and the macroscopic system (1.4), without viewing both systems as issued from a same fully discrete system (Godunov scheme) with different limits, and without passing in Lagrangian coordinates. For related works, including vacuum, see also [2, 17, 18].

The present paper is arranged in the following way: in the next section we study the Riemann Problem for (1.4) and present the qualitative properties of this model from the point of view of traffic. In Section 3 we compare the present model with others in the current literature and in Section 4 we establish the connection with a microscopic Follow-The-Leader model based on ordinary differential equations. We also show rigorously that the macroscopic model (1.4) can be viewed as the limit of the microscopic model as the number of vehicles increases to infinity. All proofs are gathered in the last section.

\section{Notation and Main Results}

We assume throughout the following hypotheses:

a. $R, \check{w}, \hat{w}, V_{\max }$ are positive constants, with $\check{w}<\hat{w}$. 
b. $\psi \in \mathbf{C}^{2}([0, R] ;[0,1])$ is such that

$$
\begin{array}{rlrl}
\psi(0) & =1, & \psi(R) & =0 \\
\psi^{\prime}(\rho) & \leq 0, \quad \frac{d^{2}}{d \rho^{2}}(\rho \psi(\rho)) & \leq 0 \quad \text { for all } \rho \in[0, R] .
\end{array}
$$

c. $\check{w}>V_{\max }$.

Here, $R$ is the maximal possible density, typically $R=1$ if $\rho$ is normalized as in Section 4, $\check{w}$, respectively $\hat{w}$, is the minimum, respectively maximum, of the maximal speeds of each vehicle; $V_{\max }$ is the overall uniform upper bound on the traffic speed. At $\mathbf{b}$, , the first three assumptions on $\Psi$ are the classical conditions usually assumed on speed laws, while the fourth one is technically necessary in the proof of Theorem 2.1. The latter condition means that all drivers do feel the presence of the speed limit.

Moreover, we introduce the notation

$$
\begin{aligned}
& F=\left\{(\rho, w) \in[0, R] \times[\check{w}, \hat{w}]: v(\rho, \rho w)=V_{\max }\right\} \\
& C=\{(\rho, w) \in[0, R] \times[\check{w}, \hat{w}]: v(\rho, \rho w)=w \psi(\rho)\}
\end{aligned}
$$

to denote the Free and the Congested phases. Note that $F$ and $C$ are closed sets and $F \cap C \neq \emptyset$. Note also that $F$ is 1 -dimensional in the $(\rho, \rho v)$ plane
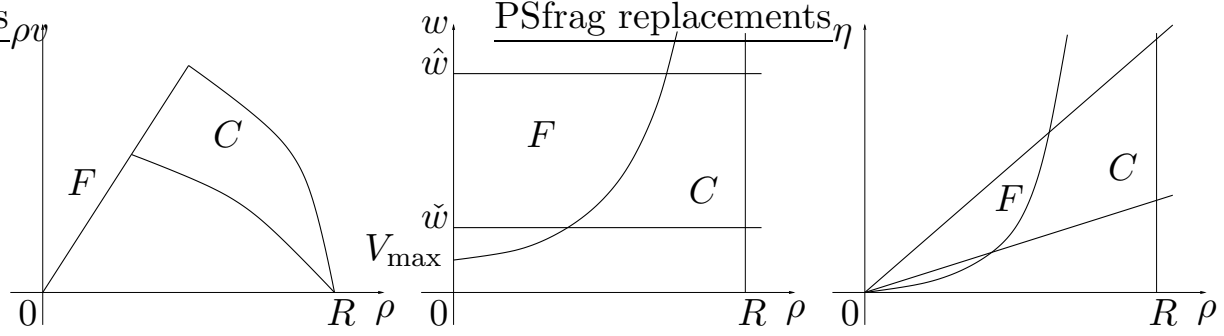

Figure 2: The phases $F$ and $C$ in the coordinates, from left to right, $(\rho, \rho v)$, $(\rho, w)$ and $(\rho, \eta)$.

of the fundamental diagram, while it is 2-dimensional in the $(\rho, w)$ and $(\rho, \eta)$ coordinates, see Figure 2. See also Figure 3 to have a vision in three dimensions.

Let $\rho_{*}$ be the maximum of the points of maximum of the flow, i.e. $\rho_{*}=$ $\max \left\{\rho \in[0, R]: \rho \psi(\rho)=\max _{r \in[0, R]} r \psi(r)\right\}$. Then, the condition

$$
\hat{w} \psi\left(\rho_{*}\right) \geq V_{\max }
$$

is a further reasonable assumption. Indeed, it means that the maximum flow is attained in the free phase, coherently with the capacity drop phenomenon, see for instance [22]. However, (2.3) is not necessary in the following results.

Our next goal is to study the Riemann Problem for (1.4). 


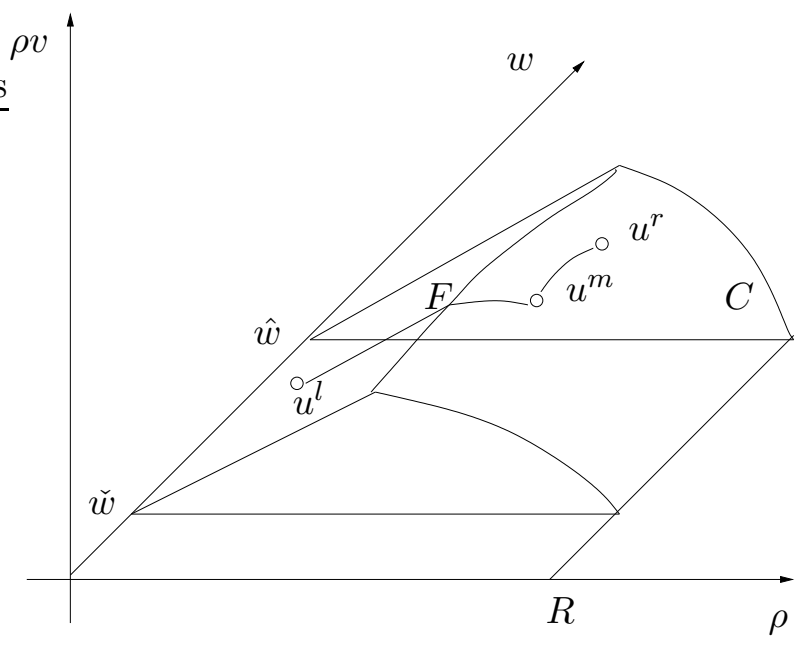

Figure 3: The phases $F$ and $C$ in the coordinates $(\rho, \rho v, w)$. Note that $F$ is contained in a plane. This figure shows an example of Riemann Problem when $u^{l}=\left(\rho^{l}, \rho^{l} v^{l}, w^{l}\right) \in F$ and $u^{r}=\left(\rho^{r}, \rho^{r} v^{r}, w^{r}\right) \in C$.

Theorem 2.1 Under the assumptions $\mathbf{a}$., $\boldsymbol{b}$. and $\mathbb{C}$., for all states $\left(\rho^{l}, \eta^{l}\right)$, $\left(\rho^{r}, \eta^{r}\right) \in F \cup C$, the Riemann problem consisting of (1.4) with initial data

$$
\rho(0, x)=\left\{\begin{array}{ll}
\rho^{l} & \text { if } x<0 \\
\rho^{r} & \text { if } x>0
\end{array} \quad \eta(0, x)= \begin{cases}\eta^{l} & \text { if } x<0 \\
\eta^{r} & \text { if } x>0\end{cases}\right.
$$

admits a unique self similar weak solution $(\rho, \eta)=(\rho, \eta)(t, x)$ constructed as follows:

(1) If $\left(\rho^{l}, \eta^{l}\right),\left(\rho^{r}, \eta^{r}\right) \in F$, then

$$
(\rho, \eta)(t, x)= \begin{cases}\left(\rho^{l}, \eta^{l}\right) & \text { if } \quad x<V_{\max } t \\ \left(\rho^{r}, \eta^{r}\right) & \text { if } \quad x>V_{\max } t .\end{cases}
$$

(2) If $\left(\rho^{l}, \eta^{l}\right),\left(\rho^{r}, \eta^{r}\right) \in C$, then $(\rho, \eta)$ consists of a 1-Lax wave (shock or rarefaction) between $\left(\rho^{l}, \eta^{l}\right)$ and $\left(\rho^{m}, \eta^{m}\right)$, followed by a 2-contact discontinuity between $\left(\rho^{m}, \eta^{m}\right)$ and $\left(\rho^{r}, \eta^{r}\right)$. The middle state $\left(\rho^{m}, \eta^{m}\right)$ is in $C$ and is uniquely characterized by the two conditions $\eta^{m} / \rho^{m}=\eta^{l} / \rho^{l}$ and $v\left(\rho^{m}, \eta^{m}\right)=v\left(\rho^{r}, \eta^{r}\right)$.

(3) If $\left(\rho^{l}, \eta^{l}\right) \in C$ and $\left(\rho^{r}, \eta^{r}\right) \in F$, then the solution $(\rho, \eta)$ consists of a rarefaction wave separating $\left(\rho^{r}, \eta^{r}\right)$ from a state $\left(\rho^{m}, \eta^{m}\right)$ and by a linear wave separating $\left(\rho^{m}, \eta^{m}\right)$ from $\left(\rho^{l}, \eta^{l}\right)$. The middle state $\left(\rho^{m}, \eta^{m}\right)$ is in $F \cap C$ and is uniquely characterized by the two conditions $\eta^{m} / \rho^{m}=\eta^{r} / \rho^{r}$ and $v\left(\rho^{m}, \eta^{m}\right)=V$. 
(4) If $\left(\rho^{l}, \eta^{l}\right) \in F$ and $\left(\rho^{r}, \eta^{r}\right) \in C$, then $(\rho, \eta)$ consists of a shock between $\left(\rho^{l}, \eta^{l}\right)$ and $\left(\rho^{m}, \eta^{m}\right)$, followed by a contact discontinuity between $\left(\rho^{m}, \eta^{m}\right)$ and $\left(\rho^{r}, \eta^{r}\right)$. The middle state $\left(\rho^{m}, \eta^{m}\right)$ is in $C$ and is uniquely characterized by the two conditions $\eta^{m} / \rho^{m}=\eta^{l} / \rho^{l}$ and $v\left(\rho^{m}, \eta^{m}\right)=$ $v\left(\rho^{r}, \eta^{r}\right)$.

(If $\frac{d^{2}}{d \rho^{2}}(\rho \psi(\rho))$ vanishes, then the words "shock" and "rarefaction" above have to be understood as "contact discontinuities").

We now pass from the solution to single Riemann problems to the properties of the Riemann Solver, i.e. of the map $\mathcal{R}:(F \cup C)^{2} \rightarrow \mathbf{B V}(\mathbb{R} ; C \cup F)$ such that $\mathcal{R}\left(\left(\rho^{l}, \eta^{l}\right),\left(\rho^{r}, \eta^{r}\right)\right)$ is the solution to (1.4) $-(\underline{2.4})$ computed at time, say, $t=1$.

To this aim, recall the following definition, see [10]:

Definition 2.2 A Riemann Solver $\mathcal{R}$ is consistent if the following two conditions hold for all $\left(\rho^{l}, \eta^{l}\right),\left(\rho^{m}, \eta^{m}\right),\left(\rho^{r}, \eta^{r}\right) \in F \cup C$, and $\bar{x} \in \mathbb{R}$ :

(C1) If $\mathcal{R}\left(\left(\rho^{l}, \eta^{l}\right),\left(\rho^{m}, \eta^{m}\right)\right)(\bar{x})=\left(\rho^{m}, \eta^{m}\right)$ and $\mathcal{R}\left(\left(\rho^{m}, \eta^{m}\right),\left(\rho^{r}, \eta^{r}\right)\right)(\bar{x})$ $=\left(\rho^{m}, \eta^{m}\right)$, then

$$
\mathcal{R}\left(\left(\rho^{l}, \eta^{l}\right),\left(\rho^{r}, \eta^{r}\right)\right)=\left\{\begin{array}{l}
\mathcal{R}\left(\left(\rho^{l}, \eta^{l}\right),\left(\rho^{m}, \eta^{m}\right)\right), \text { if } x<\bar{x}, \\
\mathcal{R}\left(\left(\rho^{m}, \eta^{m}\right),\left(\rho^{r}, \eta^{r}\right)\right), \text { if } x \geq \bar{x}
\end{array}\right.
$$

(C2) If $\mathcal{R}\left(\left(\rho^{l}, \eta^{l}\right),\left(\rho^{r}, \eta^{r}\right)\right)(\bar{x})=\left(\rho^{m}, \eta^{m}\right)$, then

$$
\begin{aligned}
& \mathcal{R}\left(\left(\rho^{l}, \eta^{l}\right),\left(\rho^{m}, \eta^{m}\right)\right)= \begin{cases}\mathcal{R}\left(\left(\rho^{l}, \eta^{l}\right),\left(\rho^{r}, \eta^{r}\right)\right), & \text { if } x \leq \bar{x}, \\
\left(\rho^{m}, \eta^{m}\right), & \text { if } x>\bar{x},\end{cases} \\
& \mathcal{R}\left(\left(\rho^{m}, \eta^{m}\right),\left(\rho^{r}, \eta^{r}\right)\right)= \begin{cases}\left(\rho^{m}, \eta^{m}\right), & \text { if } x<\bar{x}, \\
\mathcal{R}\left(\left(\rho^{l}, \eta^{l}\right),\left(\rho^{r}, \eta^{r}\right)\right), & \text { if } x \geq \bar{x} .\end{cases}
\end{aligned}
$$

Essentially, (C1) states that whenever two solutions to two Riemann problems can be placed side by side, then their juxtaposition is again a solution to a Riemann problem. Condition (C2) is the vice-versa.

The next result characterizes the Riemann Solver defined above.

Proposition 2.3 Let the assumptions $\mathbf{a}$., $\mathbf{b}$. and $\mathbb{c}$. hold. The Riemann Solver $\mathcal{R}$ defined in Theorem 2.1 enjoys the following three conditions

1. It is consistent in the sense of Definition 2.2. 


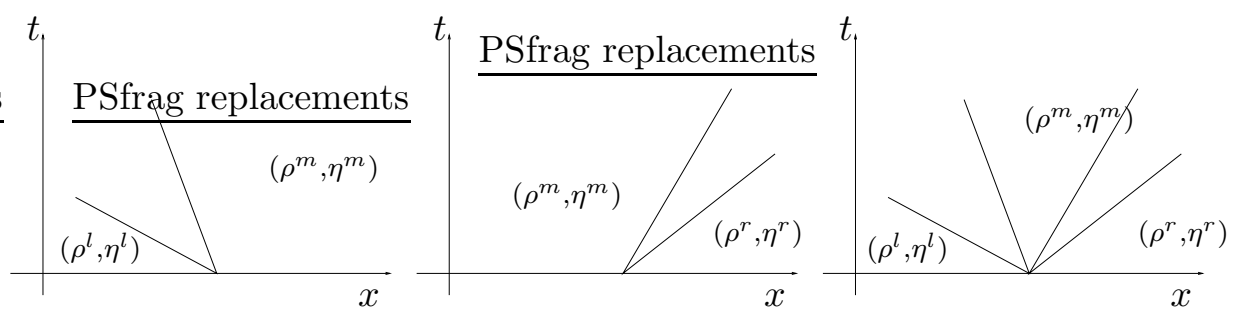

Figure 4: The conditions (C1) and (C2).

2. If $\left(\rho^{l}, \eta^{l}\right),\left(\rho^{r}, \eta^{r}\right) \in F$, then $\mathcal{R}\left(\left(\rho^{l}, \eta^{l}\right),\left(\rho^{r}, \eta^{r}\right)\right)$ is the standard solution to the linear system

$$
\left\{\begin{array}{l}
\partial_{t} \rho+\partial_{x}\left(\rho V_{\max }\right)=0 \\
\partial_{t} \eta+\partial_{x}\left(\eta V_{\max }\right)=0
\end{array}\right.
$$

3. If $\left(\rho^{l}, \eta^{l}\right) \in F \cup C$ and $\left(\rho^{r}, \eta^{r}\right) \in C$, then $\mathcal{R}\left(\left(\rho^{l}, \eta^{l}\right),\left(\rho^{r}, \eta^{r}\right)\right)$ is the standard Lax solution to

$$
\left\{\begin{array}{l}
\partial_{t} \rho+\partial_{x}(\eta \psi(\rho))=0 \\
\partial_{t} \eta+\partial_{x}\left(\frac{\eta^{2}}{\rho} \psi(\rho)\right)=0
\end{array}\right.
$$

Moreover, the conditions (C1), 2. and 3. uniquely characterize the Riemann Solver $\mathcal{R}$.

The above properties are of use, for instance, in using model (1.4) on traffic networks, according to the techniques described in [15].

The next result presents the relevant qualitative properties of the Riemann Solver defined in Theorem 2.1 from the point of view of traffic.

Proposition 2.4 Let the assumptions $\mathbf{a}$., $\mathbf{b}$. and $\mathbf{c}$. hold. Then, the Riemann Solver $\mathcal{R}$ enjoys the following properties:

1. If the initial datum attains values in $F, C$, or $F \cup C$ then, respectively, the solution attains values in $F, C$, or $F \cup C$.

2. Traffic density and speed are uniformly bounded.

3. Traffic speed vanishes if and only if traffic density is maximal.

4. No wave in the solution to (1.4) 2.4) may travel faster than traffic speed, i.e. information is carried by vehicles. 


\section{Comparison with Other Macroscopic Models}

This section is devoted to compare the present model (1.4) with a sample of models from the literature. In particular, we consider differences in the number of free parameters and functions, in the fundamental diagram and in the qualitative structures of the solutions. Recall that the evolution described by model (1.4) and the corresponding invariant domain depends on the function $\psi$ and on the parameters $V_{\max }, R, \breve{w}$ and $\hat{w}$. The fundamental diagram of (1.4) is in Figure 2, left.

\subsection{The LWR Model}

In the LWR model (1.1), a suitable speed law has to be selected, analogous to the choice of $\psi$ in (1.4). Besides, in (1.4) we also have to set $V_{\max }, R$ and the two geometric positive parameters $\check{w}$ and $\hat{w}$.

The fundamental diagram of (1.4) seems to better agree with experimental data than that of (1.1), shown in Figure 5, left. Indeed, compare
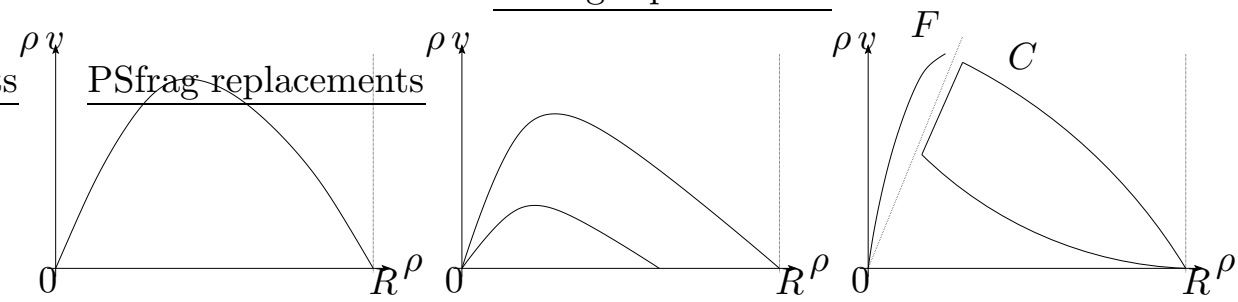

Figure 5: Fundamental diagrams, from left to right, of the (LWR) model (1.1), of the (AR) model (3.1) and of the 2-phase model (3.2).

Figure 2, left with the measurements in Figure 1.

As long as the data are in $F$, the solutions to (1.4) are essentially the same as those of (1.1). In the congested phase, the solutions to (1.4) obviously present a richer structure, for they generically contain 2 waves instead of 1 . In particular, the (LWR) model (1.1) may not describe the "homogeneousin-speed" solutions, i.e. a type of synchronized flow, see [26, Section 2.2] and [23, 37], which is described by the 2 -waves in (1.4).

Finally, note that if in (1.4) the two geometric parameters $\check{w}$ and $\hat{w}$ coincide, then we recover the LWR (1.1) model with $V(\rho)=\min \left\{V_{\max }, \hat{w} \psi(\rho)\right\}$.

\subsection{The Aw-Rascle Model}

Consider now the Aw-Rascle (AR) model

$$
\left\{\begin{array}{l}
\partial_{t} \rho+\partial_{x}[\rho v(\rho, y)]=0 \\
\partial_{t} y+\partial_{x}[y v(\rho, y)]=0
\end{array} \quad v(\rho, y)=\frac{y}{\rho}-p(\rho)\right.
$$


introduced in [4] and successively refined in several papers, see for instance [3, 6, 16, 18, 19, 20, 24, 33, 35] and the references therein.

Note that $w$ in (1.4) plays a role analogous to that of $v+p(\rho)$ in (3.1).

In the (AR) model, $R$ and the "pressure" function need to be selected, similarly to $R$ and $\psi$ in (1.4). No other parameter appears in (3.1), but the definition of an invariant domain requires two parameters, with a role similar to that of $\check{w}$ and $\hat{w}$. Indeed, an invariant domain for (3.1) is

$$
\left\{(\rho, y): \rho \in[0, R] \text { and } y \in\left[\rho\left(v_{-}+p(\rho)\right), \rho\left(v_{-}+p(\rho)\right)\right]\right\}
$$

see Figure 5, center, and depends on the speeds $v_{-}$and $v_{+}$. More recent versions of (3.1) contain also a suitable relaxation source term in the right hand side of the second equation; in this case one more arbitrary function needs to be selected. The original (AR) model does not distinguish between a free and a congested phase. However, it was extended to describe two different phases in [16]. Further comments on (3.1) are found in [29].

Concerning the analytical properties of the solutions, the Riemann solver for the (AR) model suffers lack of continuous dependence at vacuum, see 4, Section 4]. However, existence of solutions attaining also the vacuum state was proved in [18, while the 2-phase construction in [16] also displays continuous dependence.

A qualitative difference between the (AR) model and the present one is property 3. in Proposition 2.4. Indeed, solutions to (3.1) may well have zero speed while being at a density strictly lower than the maximal one.

\subsection{The Hyperbolic 2-Phase Model}

Recall the model presented in [10], with a notation similar to the present one:

$$
\begin{array}{ll}
\text { Free flow: }(\rho, q) \in F, & \text { Congested flow: }(\rho, q) \in C, \\
\partial_{t} \rho+\partial_{x}\left[\rho \cdot v_{F}(\rho)\right]=0, & \left\{\begin{array}{l}
\partial_{t} \rho+\partial_{x}\left[\rho \cdot v_{C}(\rho, q)\right]=0 \\
\partial_{t} q+\partial_{x}\left[\left(q-q_{*}\right) \cdot v_{C}(\rho, q)\right]=0 \\
v_{F}(\rho)=\left(1-\frac{\rho}{R}\right) \cdot V
\end{array}\right. \\
v_{C}(\rho, q)=\left(1-\frac{\rho}{R}\right) \cdot \frac{q}{\rho}
\end{array}
$$

the phases being defined as

$$
\begin{aligned}
& F=\left\{(\rho, q) \in[0, R] \times \mathbb{R}^{+}: v_{f}(\rho) \geq V_{f}, q=\rho \cdot V\right\} \\
& C=\left\{(\rho, q) \in[0, R] \times \mathbb{R}^{+}: v_{c}(\rho . q) \leq V_{c}, \frac{q-q_{*}}{\rho} \in\left[\frac{Q_{1}-q_{*}}{R}, \frac{Q_{2}-q_{*}}{R}\right]\right\} .
\end{aligned}
$$

In (3.2) no function can be selected, on the other hand the evolution depends on the parameters $V, R$ and $q_{*}$ while the invariant domains $F$ and $C$ depend on $V_{f}, V_{c}, Q_{1}$ and $Q_{2}$. A geometric construction of the solutions to (3.2) in the congested phase is in [31. 
The main difference between fundamental diagrams of (3.2), see Figure 5. right, and that of (1.4) is that (3.2) requires the two phases to be disconnected: there is a gap between the free and the congested phase. This restriction is necessary for the well posedness of the Riemann problem for (3.2) and can be hardly justified on the basis of experimental data. More recently, the global well-posedness of the model (3.2) was proved in [11.

Note that in both models, as well as in that presented in [16], the free phase is one dimensional, while the congested phase is bidimensional.

The model (3.2) allows for the description of wide jams, i.e. of persistent waves in the congested phase moving at a speed different from that of traffic. Here, as long as $\frac{d^{2}}{d \rho^{2}}(\rho \psi(\rho))<0$, persistent phenomena can be described only through waves of the second family, which move at the mean traffic speed. We refer to [29] for further discussions on (3.2) and comparisons with other macroscopic models.

\subsection{A Kinetic Model}

Recall, with a notation adapted to the present case, the kinetic model introduced in [8, Section 1]:

$$
\partial_{t} r(t, x ; w)+\partial_{x}\left[w r(t, x ; w) \psi\left(\int_{\breve{w}}^{\hat{w}} r\left(t, x ; w^{\prime}\right) d w^{\prime}\right)\right]=0 .
$$

The function $\psi$ and the speed $w$ play the same role as here. The unknown $r=r(t, x ; w)$ is the probability density of vehicles having maximal speed $w$ that at time $t$ are at point $x$.

In (3.3) there is one function to be specified, $\psi$, as in (1.4). The parameters are $R$ (which is normalized to 1 in [8]), $\check{w}$ and $\hat{w}$, similarly to (1.4). Since no limit speed is there defined, no parameter in (3.3) has the same role as here $V_{\max }$.

Being of a kinetic nature, there is no real equivalent to a fundamental diagram for (3.3).

From the analytical point of view, the existence of solutions to (3.3) has not been proved, yet. The main result in [8] only states that (3.3) can be rigorously obtained as the limit of systems of $n \times n$ conservation laws describing $n$ populations of vehicles, each characterized by their maximal speed.

Let the measure $r$ solve (3.3) and be such that for suitable functions $\rho$ and $w$

$$
r(t, x ; \cdot)=\rho(t, x) \delta_{w(t, x)}
$$

where $\delta$ is the usual Dirac measure. Then, formally, $(\rho, w)$ solves (1.4). Indeed, for the first equation simply substitute (3.4) in (3.3) and integrate; for the second equation substitute (3.4) in (3.3), multiply by $w$ and integrate over $[\check{w}, \hat{w}]$. 
Remark that (3.4) suggests a further interpretation of the quantity $\rho$ in (1.4). Indeed, in the present model, at $(t, x)$ vehicles of only one species are present, namely those with maximal speed $w(t, x)$.

\section{Connections with a Follow-The-Leader Model}

Within the framework of (1.3), a single driver starting from $\tilde{p}$ at time $t=0$ follows the particle path $p=p(t)$ that solves the Cauchy problem

$$
\left\{\begin{array}{l}
\dot{p}=v\left(\rho(t, p(t)), w((t, p(t))) \quad v(\rho, w)=\min \left\{V_{\max }, w \psi(\rho)\right\},\right. \\
p(0)=\tilde{p}
\end{array}\right.
$$

refer to [12 for the well posedness of the particle path for the LWR model (see also [3]). Recall now that $w$ is a specific feature of every single driver, i.e. $w(t, p(t))=w(0, \tilde{p})$ for all $\tilde{p}$. On the other hand, from a microscopic point of view, if $n$ drivers are distributed along the road, then $\rho$ is approximated by $l /\left(p_{i+1}-p_{i}\right)$, where $l$ is a standard length of a car.

We fix $L>0$ and assume that $n$ drivers are distributed along $[-L, L]$. Then, the natural microscopic counterpart to (1.4) is therefore the FollowThe-Leader (FTL) model defined by the Cauchy problem

$$
\begin{cases}\dot{p}_{i}=v\left(\frac{l}{p_{i+1}-p_{i}}, w_{i}\right) & i=1, \ldots, n \\ \dot{p}_{n+1}=V_{\max } & i=1, \ldots, n+1 \\ p_{i}(0)=\tilde{p}_{i} & i=1\end{cases}
$$

where $\tilde{p}_{1}=-L$ and $\tilde{p}_{n+1}=L-l$. Proposition 4.1 shows that (4.2) admits a unique global solution defined for every $t \geq 0$ and such that $p_{i+1}-p_{i} \geq l$ for all $t \geq 0$.

Proposition 4.1 Let a., b. and $\boldsymbol{c}$. hold. Fix $L>0$. For any $n \in \mathbb{N}$, with $n \geq 2$, choose initial data $\tilde{p}_{i}^{n}$ for $i=1, \ldots, n$ satisfying $\tilde{p}_{i+1}^{n}-\tilde{p}_{i}^{n} \geq l$. Then, the Cauchy problem (4.2) admits a unique solution $p_{i}^{n}=p_{i}^{n}(t)$, for $i=1, \ldots, n+1$, defined for all $t \geq 0$ and satisfying $p_{i+1}^{n}(t)-p_{i}^{n}(t) \geq l$ for all $t \geq 0$ and for $i=1, \ldots, n$.

The proof is postponed to Section 5 .

Our next aim is to rigorously show that in the limit $n \rightarrow+\infty$ with $n l=$ constant $>0$, the microscopic model in (4.2) yields the macroscopic one in (1.4). Given the position $p^{i}$ of every single vehicle and its maximal speed $w_{i}$, for $i=1, \ldots, n+1$, the macroscopic variables $\rho, w$ are given by

$$
\rho(x)=\sum_{i=1}^{n} \frac{l}{p_{i+1}^{n}-p_{i}^{n}} \chi_{\left[p_{i}^{n}, p_{i+1}^{n}[\right.}(x) \quad \text { and } \quad w(x)=\sum_{i=1}^{n} w_{i}^{n} \chi_{\left[p_{i}^{n}, p_{i+1}^{n}[\right.}(x) .
$$


Note that necessarily $p_{i+1}^{n}-p_{i}^{n} \geq l$.

On the contrary, given $(\rho, w) \in\left(\mathbf{L}^{\mathbf{1}} \cap \mathbf{B V}\right)(\mathbb{R} ;[0,1] \times[\check{w}, \hat{w}])$, with supp $\rho$, $\operatorname{supp} w \subseteq[-L, L]$, we reconstruct a microscopic description defining $l=$ $\left(\int_{\mathbb{R}} \rho(x) d x\right) / n$ and

$$
\begin{aligned}
p_{n+1}^{n} & =L-l \\
p_{i}^{n} & =\max \left\{p \in[-L, L]: \int_{p}^{p_{i+1}} \rho(x) d x=l\right\} \quad \text { for } i=1, \ldots, n \\
w_{i}^{n} & =w\left(p_{i}^{n}+\right) \quad \text { for } i=1, \ldots, n+1 .
\end{aligned}
$$

Note that $\int_{\mathbb{R}} \rho(x) d x=n l>0$. Now we are able to rigorously show that, as the number of vehicles increases to infinity, the microscopic model in (4.2) yields the macroscopic one in (1.4).

Proposition 4.2 Let a., b. and $\boldsymbol{c}$. hold. Fix $T>0$. Choose $(\tilde{\rho}, \tilde{w}) \in$ $\left(\mathbf{L}^{\mathbf{1}} \cap \mathbf{B V}\right)(\mathbb{R} ;[0,1] \times[\check{w}, \hat{w}])$ with $\operatorname{supp} \tilde{\rho}, \operatorname{supp} \tilde{w} \subseteq[-L, L]$. Construct the initial data for the microscopic model setting $l=\left(\int_{\mathbb{R}} \tilde{\rho}(x) d x\right) / n$ and

$$
\begin{aligned}
\tilde{p}_{n+1}^{n} & =L-l \\
\tilde{p}_{i}^{n} & =\max \left\{p \in[-L, L]: \int_{p}^{\tilde{p}_{i+1}} \tilde{\rho}(x) d x=l\right\} \quad \text { for } i=1, \ldots, n \\
\tilde{w}_{i}^{n} & =\tilde{w}\left(p_{i}^{n}+\right) \quad \text { for } i=1, \ldots, n+1 .
\end{aligned}
$$

Let $p_{i}^{n}(t)$, for $i=1, \ldots, n$, be the corresponding solution to (4.2). Define

$$
\begin{aligned}
\rho^{n}(t, x) & =\sum_{i=1}^{n} \frac{l}{p_{i+1}^{n}(t)-p_{i}^{n}(t)} \chi_{\left[p_{i}^{n}(t), p_{i+1}^{n}(t)[\right.}(x) \\
w^{n}(t, x) & =\sum_{i=1}^{n} \tilde{w}_{i}^{n} \chi_{\left[p_{i}^{n}(t), p_{i+1}^{n}(t)[\right.}(x) .
\end{aligned}
$$

If there exists a pair $(\rho, w) \in \mathbf{L}^{\infty}\left([0, T] ; \mathbf{L}^{\mathbf{1}}(\mathbb{R} ;[0,1] \times[\check{w}, \hat{w}])\right.$ such that

$$
\lim _{n \rightarrow+\infty}\left(\rho^{n}, w^{n}\right)(t, x)=(\rho, w)(t, x) \quad \text { p.a.e. }
$$

then, the pair $(\rho, \rho w)$ is a weak solution to 1.4) with initial datum $(\tilde{\rho}, \tilde{\rho} \tilde{w})$.

The proof is postponed to Section 5 .

\section{Technical Details}

We first prove an elementary consequence of our assumption b. 
Lemma 5.1 Let $\psi$ satisfy $b$. Then,

$$
\exists \bar{\rho} \in\left[0, R\left[\text { such that } \left\{\begin{array}{l}
\psi \text { is constant on }[0, \bar{\rho}], \\
\psi \text { is strictly decreasing on }[\bar{\rho}, R] .
\end{array}\right.\right.\right.
$$

Proof. Call $q(\rho)=\rho \psi(\rho)$. If $\psi$ is strictly monotone, then $\bar{\rho}=0$ and the proof is completed. Otherwise, assume that $\psi\left(\rho_{1}\right)=\psi\left(\rho_{2}\right)=c$ for suitable $\left.\left.\rho_{1}, \rho_{2} \in\right] 0, R\right]$ and $\rho_{1} \neq \rho_{2}$. Then, by $\mathbf{b}$, for all $\rho \in\left[\rho_{1}, \rho_{2}\right]$ we have $\psi(\rho)=c$ and $q(\rho)=c \rho$. If $\psi(0)=c$, then the proof is completed. Otherwise, note that $q^{\prime}(0)=\psi(0)>c$ contradicts the convexity of $q$.

Corollary $\mathbf{5 . 2}$ Let $\psi$ satisfy $[\mathbf{b}$. and $\underline{\mathbf{C}}$. Then,

$$
\bar{\rho}<\min \{\rho \in[0, R]: \exists w \in[\check{w}, \hat{w}] \text { such that }(\rho, w) \in C\} .
$$

The proof is immediate and, hence, omitted.

In the sequel, for the basic definitions concerning the standard theory of conservation laws we refer to [9].

Proof of Theorem 2.1. We consider different cases, depending on the phase of the data (2.4).

1. $\left(\rho^{l}, \eta^{l}\right),\left(\rho^{r}, \eta^{r}\right) \in F$.

In this case, (1.4) reduces to the degenerate linear system (2.6) so that the problem (1.4)-(2.4) is solved by (2.5). Remark, for later use, that the characteristic speed is $\lambda^{F}=V_{\max }$.

2. $\left(\rho^{l}, \eta^{l}\right),\left(\rho^{r}, \eta^{r}\right) \in C$.

Now, $v(\rho, \eta)=\eta \psi(\rho) / \rho$. We show that $C$ is invariant with respect to the $2 \times 2$ system of conservation laws (2.7). To this aim, we compute the eigenvalues, right eigenvectors and the Lax curves in $C$ :

$$
\begin{aligned}
\lambda_{1}(\rho, \eta) & =\eta \psi^{\prime}(\rho)+v(\rho, \eta) & \lambda_{2}(\rho, \eta) & =v(\rho, \eta) \\
r_{1}(\rho, \eta) & =\left[\begin{array}{c}
-\rho \\
-\eta
\end{array}\right] & r_{2}(\rho, \eta) & =\left[\begin{array}{c}
1 \\
\eta\left(\frac{1}{\rho}-\frac{\psi^{\prime}(\rho)}{\psi(\rho)}\right)
\end{array}\right] \\
\nabla \lambda_{1} \cdot r_{1} & =-\frac{d^{2}}{d \rho^{2}}[\rho \psi(\rho)] & \nabla \lambda_{2} \cdot r_{2} & =0 \\
\mathcal{L}_{1}\left(\rho ; \rho_{o}, \eta_{o}\right) & =\eta_{o} \frac{\rho}{\rho_{o}} & \mathcal{L}_{2}\left(\rho ; \rho_{o}, \eta_{o}\right) & =\frac{\rho v\left(\rho_{o}, \eta_{o}\right)}{\psi(\rho)}, \rho_{o}<R .
\end{aligned}
$$

When $\rho_{o}=R$, the $2-$ Lax curve through $\left(\rho_{o}, \eta_{o}\right)$ is the segment $\rho=R$, $\eta \in[R \check{w}, R \hat{w}]$.

Shock and rarefaction curves of the first characteristic family coincide by [5, Lemma 2.1], see also [9, Problem 1, Chapter 5]. The second characteristic field is linearly degenerate. Hence, (2.7) is a Temple system and $C$ is invariant, since its boundary consists of Lax curves, see [25, Theorem 3.2]. 
Thus, the solution to (1.4) is as described in (2) and attains values in $C$. 3. $\left(\rho^{l}, \eta^{l}\right) \in C,\left(\rho^{r}, \eta^{r}\right) \in F$.

Let $\rho^{m}$ satisfy $\psi\left(\rho^{m}\right)=V_{\max } \rho^{r} / \eta^{r}$. Note that such $\rho^{m}$ exists in $] 0,1[$ by $\mathbf{b}$ and $\mathbf{C}$. , it is unique by Corollary 5.2. Define $\eta^{m}=\left(\rho^{m} / \rho^{r}\right) \eta^{r}$ and note that $\left(\rho^{l}, \eta^{l}\right),\left(\rho^{m}, \eta^{m}\right)$ are connected by a 1 -rarefaction wave of (2.7) having maximal speed of propagation $\lambda_{1}\left(\rho^{m}, \eta^{m}\right)<V_{\max }$. Hence, a linear wave, solution to (2.6), can be juxtaposed connecting $\left(\rho^{m}, \eta^{m}\right)$ to $\left(\rho^{l}, \eta^{l}\right)$ and the solution to (1.4) is as described at (3).

4. $\quad\left(\rho^{l}, \eta^{l}\right) \in F,\left(\rho^{r}, \eta^{r}\right) \in C$ (see Figure 3).

Note that system (2.7) can be considered on the whole of $F \cup C$. Also this set is invariant for (2.7), by [25, Theorem 3.2]. Then, in this case, we let $(\rho, \eta)$ be the standard Lax solution to (2.7), as described at (4).

Proof of Proposition 2.3. We consider different cases depending on the phase of the data (2.4).

If $\left(\rho^{l}, \eta^{l}\right),\left(\rho^{r}, \eta^{r}\right) \in F$, then $\mathcal{R}\left(\left(\rho^{l}, \eta^{l}\right),\left(\rho^{r}, \eta^{r}\right)\right)$ coincides with the Riemann solver of a linear system, which satisfies (C1). Condition (C2) is immediate since no nontrivial middle state is available.

Similarly, if $\left(\rho^{l}, \eta^{l}\right),\left(\rho^{r}, \eta^{r}\right) \in C$, then $\mathcal{R}\left(\left(\rho^{l}, \eta^{l}\right),\left(\rho^{r}, \eta^{r}\right)\right)$ coincides with the standard Riemann solver of a $2 \times 2$ system, which is consistent. The consistency of $\mathcal{R}$ then follows by the invariance of $C$, by $\mathbf{2}$. in the proof of Theorem 2.1.

By the same argument, also the case $\left(\rho^{l}, \eta^{l}\right) \in F$ and $\left(\rho^{r}, \eta^{r}\right) \in C$ is proved. Indeed, in (C2), note that the only possible nontrivial middles states are in $C$.

Finally, if $\left(\rho^{l}, \eta^{l}\right) \in C$ and $\left(\rho^{r}, \eta^{r}\right) \in F$, then $\mathcal{R}\left(\left(\rho^{l}, \eta^{l}\right),\left(\rho^{r}, \eta^{r}\right)\right)$ takes values in $F \cup C$ and is the juxtaposition of 2 consistent Riemann problems, hence (C1) holds. Concerning (C2), note that the the only possible nontrivial middles states are in $C$, and (C2) follows by the consistency of the standard Riemann solver for (2.7).

Thus 1. is proved. Assertions 2. and 3. are immediate consequences of the construction of Theorem 2.1.

Assume now that $\mathcal{R}$ satisfies 2 and 3. Then all Riemann problems with data $\left(\rho^{l}, \eta^{l}\right),\left(\rho^{r}, \eta^{r}\right) \in F,\left(\rho^{l}, \eta^{l}\right) \in F,\left(\rho^{r}, \eta^{r}\right) \in C$ and $\left(\rho^{l}, \eta^{l}\right),\left(\rho^{r}, \eta^{r}\right) \in C$ are uniquely solved. The solution to Riemann problems with $\left(\rho^{l}, \eta^{l}\right) \in C$ and $\left(\rho^{r}, \eta^{r}\right) \in F$ is then uniquely constructed through (C1).

Proof of Proposition 2.4. Consider the different statements separately.

1. The invariance of $F, C$ and $F \cup C$ is shown in the proof of Theorem 2.1 ,

2. By the invariance of $F \cup C$, it is sufficient to observe that on the compact set $F \cup C$, the density $\rho$, respectively the speed $v$, is uniformly bounded by $R$, respectively $V_{\max }$. 
3. It is immediate, see for instance Figure 2, left.

4. In phase $C$ we have

$$
\lambda_{1}(\rho, \eta)=\eta \psi^{\prime}(\rho)+v(\rho, \eta) \leq v(\rho, \eta) \quad \text { and } \quad \lambda_{2}(\rho, \eta) \leq v(\rho, \eta) .
$$

In the free phase the wave speed is $V_{\max }=v(\rho, \eta)$. The only case left is that of a phase boundary with left state in $F$ and right state, say $\left(\rho^{r}, \eta^{r}\right)$, in $C$. Then, the speed $\Lambda$ of the phase boundary clearly satisfies $\Lambda \leq \lambda_{1}\left(\rho^{r}, \eta^{r}\right)<$ $v\left(\rho^{r}, \eta^{r}\right)$.

Proof of Proposition 4.1. Note first that the functions $\rho \rightarrow v\left(\rho, w_{i}\right)$ in (4.2) are uniformly bounded and Lipschitz continuous for $i=1, \ldots, n$. We extend them to functions with the same properties and defined on $[0,+\infty[$ setting

$$
u_{i}(\rho)=\left\{\begin{array}{llc}
V_{\max } & \text { if } & \rho<0 \\
v\left(\rho, w_{i}\right) & \text { if } & \rho \in[0,1] \\
0 & \text { if } & \rho>1 .
\end{array}\right.
$$

We also note that, for $i=1, \ldots, n$, the composite applications $\delta \rightarrow u_{i}(l / \delta)$, can be extended to uniformly bounded and Lipschitz continuous functions on $[0,+\infty[$. Now we consider the Cauchy problem

$$
\begin{cases}\dot{p}_{i}^{n}=u_{i}\left(\frac{l}{p_{i+1}^{n}-p_{i}^{n}}\right) & i=1, \ldots, n \\ \dot{p}_{n+1}^{n}=V_{\max } & i=1, \ldots, n+1 . \\ p_{i}^{n}(0)=\tilde{p}_{i}\end{cases}
$$

Note that $\tilde{p}_{i}^{n}$, for $i=1, \ldots, n+1$ are defined in Proposition 4.2 and satisfy the condition $\tilde{p}_{i+1}^{n}-\tilde{p}_{i}^{n} \geq l>0$, for every $i=1, \ldots, n$.

By the standard ODE theory, there exists a $\mathbf{C}^{\mathbf{1}}$ solution $p_{i}^{n}$ defined as long as $p_{i+1}^{n}-p_{i}^{n}>0$ for all $i=1, \ldots, n$. We now prove that in fact $p_{i+1}^{n}(t)-p_{i}^{n}(t) \geq l$ for every $t \geq 0$. To this aim we assume by contradiction that there exist positive $\bar{t}$ and $t^{*}$, with $\bar{t}<t^{*}$, such that $p_{i+1}^{n}(\bar{t})-p_{i}^{n}(\bar{t})=l$ and $p_{i+1}^{n}(t)-p_{i}^{n}(t)<l$ for every $\left.\left.t \in\right] \bar{t}, t^{*}\right]$. Then,

$$
p_{i}^{n}(t)=p_{i}^{n}(\bar{t})+\int_{\bar{t}}^{t} \dot{p}_{i}(s) d s=p_{i}^{n}(\bar{t})+\int_{\bar{t}}^{t} u_{i}\left(\frac{l}{p_{i+1}^{n}(s)-p_{i}^{n}(s)}\right) d s=p_{i}^{n}(\bar{t}) .
$$

This yields a contradiction, since for every $\left.t \in] \bar{t}, t^{*}\right]$

$$
p_{i+1}^{n}(t)-p_{i}^{n}(t) \geq p_{i+1}^{n}(\bar{t})-p_{i}^{n}(\bar{t})=l,
$$

completing the proof. 
Proof of Proposition 4.2. Recall first the definition of weak solution to (1.4): for all $\varphi \in \mathbf{C}_{\mathbf{c}}^{\infty}$, setting $v(\rho, w)=\min \left\{V_{\max }, w \psi(\rho)\right\}$,

$$
\int_{0}^{T} \int_{\mathbb{R}}\left(\left[\begin{array}{c}
\rho \\
\rho w
\end{array}\right] \partial_{t} \varphi+\left[\begin{array}{c}
\rho v(\rho, w) \\
\rho w v(\rho, w)
\end{array}\right] \partial_{x} \varphi\right) d x d t+\int_{\mathbb{R}}\left[\begin{array}{c}
\tilde{\rho} \\
\tilde{\rho} \tilde{w}
\end{array}\right] \varphi(0, x) d x=0
$$

and consider the two components separately.

Below, $\mathcal{O}(1)$ denotes a constant that uniformly bounds from above the modulus of $\varphi$ and all its derivatives up to the second order. Insert first (4.3) in the above equality and obtain:

$$
\begin{aligned}
I^{n}:= & \int_{0}^{T} \int_{\mathbb{R}}\left(\rho^{n} \partial_{t} \varphi+\rho^{n} v\left(\rho^{n}, w^{n}\right) \partial_{x} \varphi\right) d x d t+\int_{\mathbb{R}} \tilde{\rho} \varphi(0, x) d x \\
= & \sum_{i=1}^{n} \int_{0}^{T} \frac{l}{p_{i+1}^{n}(t)-p_{i}^{n}(t)} \int_{p_{i}^{n}(t)}^{p_{i+1}^{n}(t)}\left[\partial_{t} \varphi+v\left(\frac{l}{p_{i+1}^{n}(t)-p_{i}^{n}(t)}, w_{i}^{n}\right) \partial_{x} \varphi\right] d t \\
& \quad+\int_{\mathbb{R}} \rho^{n}(0, x) \varphi(0, x) d x+\int_{\mathbb{R}}\left(\tilde{\rho}-\rho^{n}(0, x)\right) \varphi(0, x) d x \\
= & \sum_{i=1}^{n} \int_{0}^{T} \frac{l}{p_{i+1}^{n}(t)-p_{i}^{n}(t)} \int_{p_{i}^{n}(t)}^{p_{i+1}^{n}(t)}\left(\partial_{t} \varphi(t, x)+\dot{p}_{i}^{n}(t) \partial_{x} \varphi(t, x)\right) d x d t \\
& \quad+\sum_{i=1}^{n} \frac{l}{\tilde{p}_{i+1}^{n}-\tilde{p}_{i}^{n}} \int_{\tilde{p}_{i}}^{\tilde{p}_{i+1}} \varphi(0, x) d x+\int_{\mathbb{R}}\left(\tilde{\rho}-\rho^{n}(0, x)\right) \varphi(0, x) d x .
\end{aligned}
$$

Approximating $\varphi(t, x)$ with $\varphi\left(t, p_{i}^{n}(t)\right)$ for every $x$ in $\left[p_{i}^{n}(t), p_{i+1}^{n}(t)\right]$, we obtain:

$$
\begin{aligned}
I^{n}= & \sum_{i=1}^{n} \int_{0}^{T} \frac{l}{p_{i+1}^{n}(t)-p_{i}^{n}(t)} \int_{p_{i}^{n}(t)}^{p_{i+1}^{n}(t)} \frac{d}{d t} \varphi\left(t, p_{i}^{n}(t)\right) d x d t \\
& +\sum_{i=1}^{n} \int_{0}^{T} \frac{l}{p_{i+1}^{n}(t)-p_{i}^{n}(t)} \int_{p_{i}^{n}(t)}^{p_{i+1}^{n}(t)} \mathcal{O}(1)\left(p_{i+1}^{n}(t)-p_{i}^{n}(t)\right) d x d t \\
& +\sum_{i=1}^{n} \frac{l}{\tilde{p}_{i+1}^{n}-\tilde{p}_{i}^{n}} \int_{\tilde{p}_{i}}^{\tilde{p}_{i+1}} \varphi(0, x) d x+\int_{\mathbb{R}}\left(\tilde{\rho}-\rho^{n}(0, x)\right) \varphi(0, x) d x \\
= & l \sum_{i=1}^{n} \int_{0}^{T} \frac{d}{d t} \varphi\left(t, p_{i}^{n}(t)\right) d t+\Delta x \sum_{i=1}^{n} \int_{0}^{T} \mathcal{O}(1)\left(p_{i+1}^{n}(t)-p_{i}^{n}(t)\right) d x d t \\
& +\sum_{i=1}^{n} \frac{l}{\tilde{p}_{i+1}^{n}-\tilde{p}_{i}^{n}} \int_{\tilde{p}_{i}}^{\tilde{p}_{i+1}} \varphi(0, x) d x+\int_{\mathbb{R}}\left(\tilde{\rho}-\rho^{n}(0, x)\right) \varphi(0, x) d x \\
= & \sum_{i=1}^{n} \frac{l}{\tilde{p}_{i+1}^{n}-\tilde{p}_{i}^{n}} \int_{\tilde{p}_{i}}^{\tilde{p}_{i+1}}\left[\varphi(0, x)-\varphi\left(0, \tilde{p}_{i}^{n}\right)\right] d x \\
& +\mathcal{O}(1) l\left(p_{n+1}^{n}(T)-p_{1}^{n}(T)\right)+\int_{\mathbb{R}}\left(\tilde{\rho}-\rho^{n}(0, x)\right) \varphi(0, x) d x
\end{aligned}
$$




$$
=\mathcal{O}(1) l\left(2 L+V_{\max } T\right)+\int_{\mathbb{R}}\left(\tilde{\rho}-\rho^{n}(0, x)\right) \varphi(0, x) d x
$$

and both terms in the latter quantity clearly vanish as $n \rightarrow+\infty$.

The computations related to the other component are entirely similar, since $w$ is constant along any set of the form

$$
\left\{(t, x) \in[0, T] \times \mathbb{R}: x \in\left[p_{i}^{n}(t), p_{i+1}^{n}(t)[\}\right.\right.
$$

and the proof is completed.

Remark 5.3 System (1.2) is not in conservation form. As far as smooth solutions are concerned, it is equivalent to infinitely many $2 \times 2$ systems of conservation laws. Indeed, introduce a strictly monotone function $f \in$ $\mathbf{C}^{2}([\check{w}, \hat{w}] ;] 0,+\infty[)$. Then, elementary computations show that, as long as smooth solutions are concerned, system (1.2) is equivalent to

$$
\left\{\begin{array}{ll}
\partial_{t} \rho+\partial_{x}(\rho \psi(\rho) g(\eta / \rho))=0 \\
\partial_{t} \eta+\partial_{x}(\eta \psi(\rho) g(\eta / \rho))=0
\end{array} \quad \text { where } \quad \begin{array}{l}
\eta=\rho f(w) \text { and } \\
g(f(w))=w
\end{array}\right.
$$

Clearly, different choices of $f$ yield different weak solutions to (5.3), but they are all equivalent when written in terms of $\rho$ and $w$.

Acknowledgment. The first and second author acknowledge the warm hospitality of the Laboratoire de Mathematiques J.A. Dieudonne of the Universitè Sophia-Antipolis de Nice, where part of this work was completed.

\section{References}

[1] D. Amadori and W. Shen. Global existence of large BV solutions in a model of granular flow. Preprint, 2008.

[2] A. Aw. Modèles hyperboliques de trafic automobile. $\mathrm{PhD}$ thesis, Université de Nice, France, 2001.

[3] A. Aw, A. Klar, T. Materne, and M. Rascle. Derivation of continuum traffic flow models from microscopic follow-the-leader models. SIAM J. Appl. Math., 63(1):259-278 (electronic), 2002.

[4] A. Aw and M. Rascle. Resurrection of "second order" models of traffic flow. SIAM J. Appl. Math., 60(3):916-938 (electronic), 2000.

[5] P. Bagnerini, R. M. Colombo, and A. Corli. On the role of source terms in continuum traffic flow models. Math. Comput. Modelling, 44(9-10):917-930, 2006.

[6] P. Bagnerini and M. Rascle. A multiclass homogenized hyperbolic model of traffic flow. SIAM J. Math. Anal., 35(4):949-973 (electronic), 2003. 
[7] S. Benzoni Gavage and R. M. Colombo. An $n$-populations model for traffic flow. Europ. J. Appl. Math., 14(5):587-612, 2003.

[8] S. Benzoni-Gavage, R. M. Colombo, and P. Gwiazda. Measure valued solutions to conservation laws motivated by traffic modelling. Proc. R. Soc. Lond. Ser. A Math. Phys. Eng. Sci., 462(2070):1791-1803, 2006.

[9] A. Bressan. Hyperbolic systems of conservation laws, volume 20 of Oxford Lecture Series in Mathematics and its Applications. Oxford University Press, Oxford, 2000. The one-dimensional Cauchy problem.

[10] R. M. Colombo. Hyperbolic phase transitions in traffic flow. SIAM J. Appl. Math., 63(2):708-721, 2002.

[11] R. M. Colombo, P. Goatin, and F. S. Priuli. Global well posedness of traffic flow models with phase transitions. Nonlinear Anal., 66(11):2413-2426, 2007.

[12] R. M. Colombo and A. Marson. A Hölder continuous ODE related to traffic flow. Proc. Roy. Soc. Edinburgh Sect. A, 133(4):759-772, 2003.

[13] C. F. Daganzo. Requiem for high-order fluid approximations of traffic flow. Trans. Res., 29B(4):277-287, August 1995.

[14] L. C. Edie. Car-following and steady-state theory for noncongested traffic. Operations Res., 9:66-76, 1961.

[15] M. Garavello and B. Piccoli. Traffic flow on networks, volume 1 of AIMS Series on Applied Mathematics. American Institute of Mathematical Sciences (AIMS), Springfield, MO, 2006. Conservation laws models.

[16] P. Goatin. The Aw-Rascle vehicular traffic flow model with phase transitions. Math. Comput. Modelling, 44(3-4):287-303, 2006.

[17] M. Godvik and H. Hanche-Olsen. Car-following and the macroscopic AwRascle traffic flow model. Preprint, 2008.

[18] M. Godvik and H. Hanche-Olsen. Existence of solutions for the Aw-Rascle traffic flow model with vacuum. J. Hyperbolic Differ. Equ., 5(1):45-63, 2008.

[19] J. M. Greenberg. Extensions and amplifications of a traffic model of Aw and Rascle. SIAM J. Appl. Math., 62(3):729-745 (electronic), 2001/02.

[20] J. M. Greenberg, A. Klar, and M. Rascle. Congestion on multilane highways. SIAM J. Appl. Math., 63(3):818-833 (electronic), 2003.

[21] K. Hadeler and C. Kuttler. Dynamical models for granular matter. Granular Matter, 2(9-18), 1999.

[22] D. Helbing, A. Kesting, and M. Treiber. Understanding widely scattered traffic flows, the capacity drop, and platoons as effects of variance-driven time gaps. Physical Review E (Statistical, Nonlinear, and Soft Matter Physics), 74(1):016123, 2006.

[23] D. Helbing and M. Treiber. Critical discussion of synchronized flow. Cooper@tiveTr@nsport@tion Dyn@mics, 1(2.12.24), 2002.

[24] M. Herty and A. Klar. Modeling, simulation, and optimization of traffic flow networks. SIAM J. Sci. Comput., 25(3):1066-1087 (electronic), 2003. 
[25] D. Hoff. Invariant regions for systems of conservation laws. Trans. Amer. Math. Soc., 289(2):591-610, 1985.

[26] B. S. Kerner. Phase transitions in traffic flow. In D. Helbing, H. Hermann, M. Schreckenberg, and D. Wolf, editors, Traffic and Granular Flow '99, pages 253-283. Springer Verlag, 2000.

[27] B. L. Keyfitz and H. C. Kranzer. A system of nonstrictly hyperbolic conservation laws arising in elasticity theory. Arch. Rational Mech. Anal., 72(3):219$241,1979 / 80$.

[28] K. M. Kockelman. Modeling traffics flow-density relation: Accommodation of multiple flow regimes and traveler types. Transportation, 28:363-374, 2001.

[29] J. P. Lebacque. Inhomogeneous Riemann problem for the 1-phase Colombo model: graphical solutions. Preprint, November 2007.

[30] J. P. Lebacque, X. Louis, S. Mammar, B. Schnetzlera, and H. Haj-Salem. Modélisation du trafic autoroutier au second ordre. Comptes Rendus Mathematique, 346(21-22):1203-1206, November 2008.

[31] J. P. Lebacque, S. Mammar, and H. Haj-Salem. Generic second order traffic flow modelling. In Transportation and Traffic Theory: Proceedings of the 17th International Symposium on Transportation and Traffic Theory, 2007.

[32] M. J. Lighthill and G. B. Whitham. On kinematic waves. II. A theory of traffic flow on long crowded roads. Proc. Roy. Soc. London. Ser. A., 229:317-345, 1955.

[33] S. Moutari and M. Rascle. A hybrid Lagrangian model based on the Aw-Rascle traffic flow model. SIAM J. Appl. Math., 68(2):413-436, 2007.

[34] P. I. Richards. Shock waves on the highway. Operations Res., 4:42-51, 1956.

[35] F. Siebel and W. Mauser. On the fundamental diagram of traffic flow. SIAM J. Appl. Math., 66(4):1150-1162 (electronic), 2006.

[36] B. Temple. Systems of conservation laws with invariant submanifolds. Trans. Amer. Math. Soc., 280(2):781-795, 1983.

[37] R. Wang, R. Jiang, Q.-S. Wu, and M. Liu. Synchronized flow and phase separations in single-lane mixed traffic flow. Physica A: Statistical Mechanics and its Applications, 378(2):475 - 484, 2007. 